\title{
Kernos
}

Revue internationale et pluridisciplinaire de religion grecque antique

16 | 2003

Varia

\section{Discours odysséen de Protée}

Narrations traditionnelles et innovations narratologiques

\section{Menelaos Christopoulos}

\section{(2) OpenEdition}

1 Journals

\section{Édition électronique}

URL : http://journals.openedition.org/kernos/809

DOI : $10.4000 /$ kernos.809

ISSN : 2034-7871

Éditeur

Centre international d'étude de la religion grecque antique

\section{Édition imprimée}

Date de publication : 1 janvier 2003

Pagination : 35-41

ISSN : 0776-3824

\section{Référence électronique}

Menelaos Christopoulos, «Discours odysséen de Protée », Kernos [En ligne], 16 | 2003, mis en ligne le

14 avril 2011, consulté le 06 mai 2019. URL : http://journals.openedition.org/kernos/809 ; DOI :

$10.4000 /$ kernos.809 


\section{Discours odysséen de Protée}

\section{Narrations traditionnelles et innovations narratologiques}

L'objet de cet article est la nature des révélations que Ménélas obtient de Protée dans le chant IV de l'Odjssée (351-586). On étudiera plus particulièrement la fonction de ces révélations dans le schéma narratif odysséen et, en même temps, les rapports que l'on peut établir entre les révélations elles-mêmes et le personnage mythique de Protée dans le cadre religieux où il s'inscrit. Le but est de parvenir à définir les priorités narratologiques qui placent ces révélations particulières dans ce cadre religieux déterminé. Dans une première partie, on étudie la rencontre de Ménélas et de Protée telle qu'elle est décrite dans le texte de l'Odyssée. Ensuite, on essaie d'isoler une par une les révélations mêmes qu'obtient Ulysse du Viellard de la mer. Enfin, on avance quelques hypothèses interprétatives sur les données du récit qui concernent plus particulièrement le rôle de l'Égypte, le rôle d'Eidothéa dans le récit et, surtout, le rôle du récit lui-même qui transmet, par la bouche de Protée, les éléments d'un dialogue que le poète de l'Odyssée semble entretenir avec la poésie épique antérieure ou contemporaine.

\section{La rencontre de Protée et de Ménélas dans l'Odyssée}

Les renseignements fournis par Protée sont inclus dans le récit de Ménélas à Télémaque lorsque ce dernier visite Sparte pour obtenir des nouvelles de son père. Tout le récit de Ménélas sur la rencontre avec Protée trouve sa raison d'être dans l'avant-dernière révélation que fera Protée, celle qui concerne le sort d'Ulysse ${ }^{1}$. La rencontre entre Ménélas et Protée a lieu en Égypte où les bateaux de Ménélas sont portés par la tempête lor's de leur retour de Troie. Lorsqu'il s'efforce de quitter l'Égypte, Ménélas, faute de vents favorables, se retrouve immobilisé dans l'île de Pharos. C'est là, et alors que les ressources alimentaires se réduisent de façon drama-

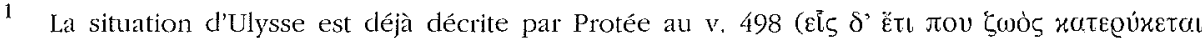

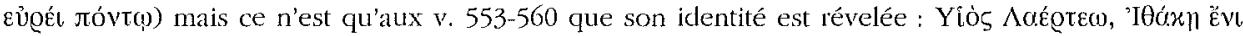

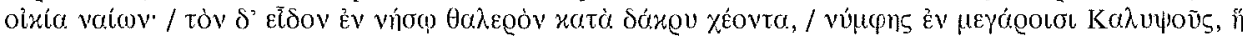

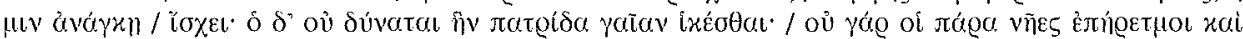

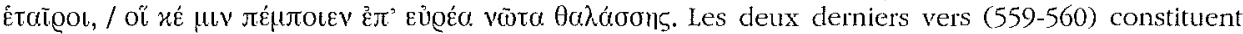
également la formule par laquelle on justifie en général les raisons qui empêchent Ulysse de quitter l'île de Calypso. Ces vers formulaires sont employés, hormis par Protée, par Athéna, lors de la cleuxième assemblée des dieux (V, 16-17), et par Calypso elle-même lors de la visite d'Hermès chez elle $(V, 141-142)$. On remarque que ces vers sont réservés à des êtres divins (Protée, Athéna, Calypso); Ulysse emploie d'autres termes (V, 173-179) pour décrire sa propre situation lorsque Calypso, au chant V (160-170), lui révèle «sa » décision (= celle des dieux) de le laisser partir et lui propose de construire un radeau pour son départ. On constate au chant $\mathrm{V}$ une tendance plus générale à distinguer nettement le niveau divin du niveau mortel; j'ai eu l'occasion d'étudier cette tendance plus en détail dans "Le départ de l'île de Calypso. Quelques remarques sur le texte de l'Odyssée", Keinos 9 (1996), p. 271-279. 
tique, que Ménélas rencontre Eidothéa, la fille de Protée. Elle lui dira comment obtenir de Protée les renseignements concernant son départ pour Sparte : couvert de peaux de phoque, avec trois compagnons, il attendra Protée au bord de la mer où le Vieillard sortira de l'eau, comme un pasteur marin, pour compter les phoques de son troupeau ${ }^{2}$. En suivant les conseils d'Eidothéa, Ménélas et ses compagnons parviennent à arrêter Protée et à le maintenir en dépit de ses six métamorphoses (en lion, serpent, panthère, sanglier, eau et arbre) ${ }^{3}$; lorsque le cycle des métamorphoses de Protée est clos, Ménélas lui pose les deux questions auxquelles il cherche une réponse: quel est le dieu qui l'empêche de partir et quelle est l'action qu'il doit entrprendre pour assurer son retour à Sparte. Protée lui fournit les réponses appropriées et on s'attendrait logiquement à voir l'entretien s'achever. Pourtant, Ménélas demande ensuite quel est le sort des autres héros grecs de la guerre de Troie; Protée met en cause l'utilité d'un tel savoir ${ }^{4}$ et limite la portée de la question en introduisant un critère spécifique pour sa réponse : il parlera des chefs des Grecs qui sont morts à la fin de leur nostos ${ }^{5}$. Ainsi les réponses données par Protée dépassent largement la portée de la question initiale tandis qu'elles réduisent celle de la seconde.

\section{Les révélations de Protée (IV, 472-569)}

Les deux premières révélations concernent effectivement le sort de Ménélas et l'action immédiate qu'il doit entreprendre pour sortir des contraintes présentes. Ménélas devait faire des offrandes en l'honneur des dieux avant son départ; c'est la raison pour laquelle les dieux ne favorisent pas son retour. Il doit à nouveau flotter sur le Nil et offrir par la suite des hécatombes aux dieux; c'est la façon par laquelle les dieux favoriseront son retour.

À partir de ce point du récit, les révélations de Protée concernent le nostos des autres héros. À la question de Ménélas sur le destin des autres Grecs, Protée répond que Ménélas ne doit pas connaître tout ce que lui connaît. Pleurer, c'est tout ce que Ménélas sera amené à faire dès qu'il aura tout su. Il fournit, néanmoins, ce qu'on pourrait considérer comme une troisième révélation, à savoir que certains d'entre eux ont péri et certains sont sauvés. La suite de son discours, il le précise, concerne non pas les Grecs en général, mais les chefs de l'armée grecque et c'est autour du retour de ces derniers qu'est placée sa quatrième révélation. Des chefs des Achéens partis pour rentrer chez eux, deux seulement sont morts et un seul vit toujours quelque part

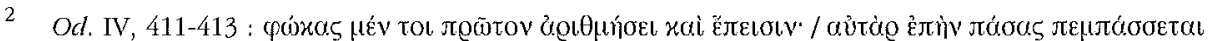

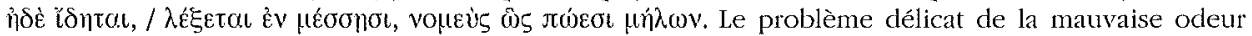

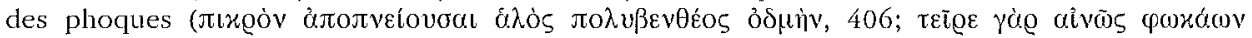

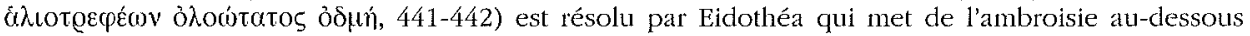
des narines des Grecs; il s'agit, en effet, d'un élément de vraisemblance destiné à renforcer la persuasion du récit et à compenser, en quelque sorte, la part du sumaturel à laquelle il est par moments assujetti.

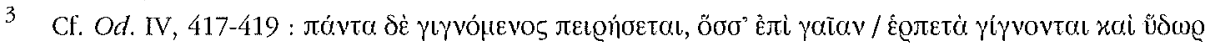

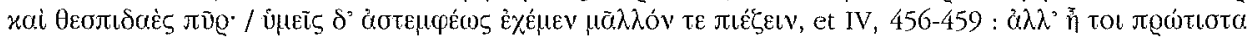

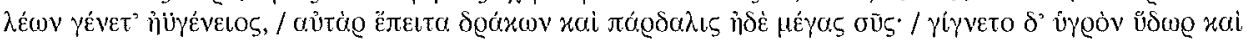

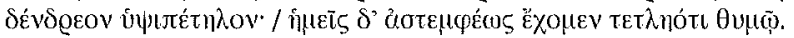

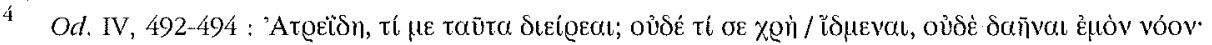

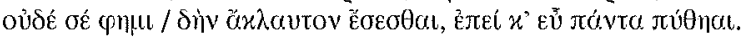

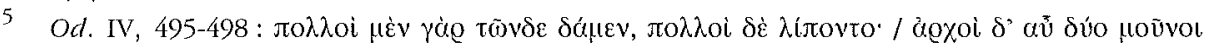

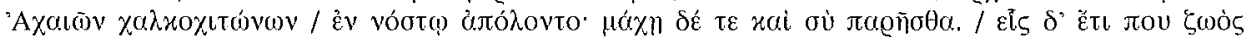

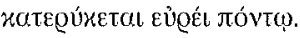


dans la mer sans pouvoir rentrer. Ainsi, les révélations suivantes traitent, tout naturellement, du sort de ces trois héros. Le premier d'entre eux, sujet de la cinquième révélation protéenne, est Ajax le Locrien. Or, d'après Protée, Ajax est mort et ses bateaux ont été détruits près des pierres Gyrées. Après le naufrage, Ajax est parvenu à s'accrocher au rocher, grâce à la tolérance de Poséidon et en dépit de l'hostilité d'Athéna. Il aurait été sauvé s'il ne s'était pas vanté de pouvoir vaincre la volonté des dieux. En entendant ces vantardises, Poséidon a précipité le rocher et Ajax s'est noyé au milieu des flots. Agamemnon est le deuxième des trois héros dont le sort est raconté par Protée. Le roi de l'armée achéenne est l'objet de la sixième et de la septième révélation de Protée. Contrairement à Ajax, Agamemnon a échappé aux dangers maritimes, grâce à l'aide d'Héra. Une tempête au Cap Malée l'a poussé vers la région où habitait Égisthe; des vents plus favorables l'ont par la suite amené chez lui. C'est là qu'il a assisté, avec ses compagnons, au banquet au cours duquel il a été traitreusement assassiné par Égisthe et ses hommes. Les auditeurs, amenés à imaginer qu'alors Ménélas s'effondrait en larmes, entendent Protée dans cette septième révélation lancer la perspective de la vengeance d'Oreste : Ménélas doit arrêter les pleurs et rentrer au plus tôt en Grèce pour rendre justice du meurtre de son frère - à moins qu'Oreste n'arrive à le faire de son côté. Ce n'est que par la huitième révélation de Protée qu'on apprendra le sort du troisième chef dont parle Protée, sort dont l'état actuel est déjà connu par les auditeurs/lecteurs de l'Odyssée, mais qui constitue le seul motif de l'évocation du récit protéen aussi bien que du voyage de Télémaque. Ce troisième chef achéen qui est toujours en vie mais qui n'est pas en mesure de poursuivre son nostos maritime est, bien entendu, Ulysse. Protée l'a vu pleurer (comme les auditeurs vont le voir au chant $V, 151-158$ ) dans l'île de Calypso qui le retient malgré lui puisqu'il n'y a pas de bateaux ni d'hommes qui lui permettraient de traverser la mer. Puisque le but du récit a été atteint, il peut alors se conclure. La neuvième et dernière révélation protéenne ramène le discours sur le sort de Ménélas tout en le prolongeant. La fin du discours adressé à Ménélas parle de la fin de sa vie. Protée lui prédit qu'il ne mourra pas à Argos; les dieux l'amèneront aux Champs Élysées où il va partager la vie sans peine des immortels grâce à son alliance avec Hélène qui fait de lui le gendre de Zeus. Ainsi se terminent les révélations obtenues de Protée, révélations qui, d'une part, permettront à Ménélas de quitter l'Égypte et qui, d'autre part, serviront en quelque sort d'« alibi » narratologique en reliant le voyage de Télémaque au nostos d'Ulysse qui est l'objet de l'épopée odysséenne mais aussi avec un récit épique qui concerne le nostos d'autres héros. Il convient alors d'interroger quelques aspects du récit odysséen sur Protée qui concernent plus particulièrement le milieu naturel dans lequel se situe la rencontre, à savoir l'Égypte, le rôle d'intermédiaire octroyé par le poète à Eidothéa et sa fonction dans la structure générale de l'Odyssée, et la valeur narratologique que reçoivent les révélations de Protée par rapport aux renseignements que le récit épique doit fournir aux auditeurs.

\section{L'Égypte}

La rencontre entre Ménélas et Protée a lieu dans l'île de Pharos située, en réalité, à une distance inférieure à un mille des côtes d'Alexandrie. La distance évoquée dans l'épopée (navigation d'un jour) présente délibérément Ménélas comme plus éloigné et plus isolé. La topographie égyptienne est identifiable, mais en même temps inconnue et inquiétante. Le nom de Pharos apparaît comme une forme hellénisée d'un toponyme égyptien $(P r-H r=$ habitation de Horus ?) et contribue, lui aussi, à la crédibilité du récit. Quoique la précision des détails topographiques ne préoccupe pas particu- 
lièrement le poète, ni n'est exigée par les connaissances spécifiques de son public, des notions générales sur l'Égypte sont assurées pour les Grecs de l'époque homérique, même si elles relèvent de l'ouï-dire ${ }^{6}$. Les rapports systématiques entre la Grèce et l'Égypte sont plus tardifs, surtout à partir du règne du roi Psammétique ${ }^{\text {er }}$ (664-610 av. J.-C.), période pendant laquelle s'opère l'installation des Grecs à Naucratis. Mais déjà pendant le IXe siècle av. J.-C., il y a des objets égyptiens en Grèce (Lefkandi). Pendant le VIII ${ }^{e}$ siècle, il y en a encore plus, même s'ils arrivent en terre grecque par Chypre et par la Phénicie ${ }^{7}$; la maîtrise des routes maritimes vers l'Égypte est attestée dans l'Odyssée, en particulier dans les récits mensongers d'Ulysse (XIV, 246-316; XVII, 424-444), où l'on mentionne également l'activité de pirates. On y signale aussi, pour la deuxième fois, la navigation sur le $\mathrm{Nil}^{8}$.

\section{Eidothéa}

Le rôle secourable d'Eidothéa qui, de son propre gré, offre ses conseils à Ménélas ne correspond pas seulement au thème du conte, à savoir celui de la fille qui aide un étranger en agissant contre son père, suivant un motif bien connu, mais il constitue aussi l'équivalent narratif de Leucothéa qui, de son propre gré elle aussi, offre son secours à Ulysse lorsque ce dernier se bat contre les flots ${ }^{9}$. Des analogies narratives délibérées entre l'errance d'Ulysse et l'errance de Ménélas ont été souvent repérées à juste titre ${ }^{10}$ (d'ailleurs, le poète lui même y fait allusion lorsqu'il intègre des parties des aventures de Ménélas dans les récits mensongers d'Ulysse). Il est donc probable que les analogies entre le nom des deux personnages (Eidothéa-Leucothéa) ne relèvent pas du hasard. Il est évident que le nom même d'Eidothéa a une signification. Toutefois, il est moins évident de percevoir la signification précise vers laquelle il oriente notre compréhension : le savoir des dieux $(\varepsilon i \delta \cup \tilde{L} \alpha)^{11}$ ? les divers aspects des dieux à métamorphoses ( $\varepsilon \grave{\delta} \delta \circ \zeta$ ) ? la beauté divine en (renversant les deux composantes de l'adjectif $\theta \varepsilon o \varepsilon t \delta n ́)^{12}$ ? Le contexte ne paraît guère favoriser une de ces interprétations au détriment des autres et peut-être impose-t-il ainsi la force accumulative des trois. Dans le drame satyrique Protée qui clôturait la tétralogie de l'Orestie, Eschyle appelle la fille de Protée Eidô (Eỉó́) $)^{13}$. Dans l'Hélène, Euripide conserve le nom d'Eidô pour la fille de Protée en précisant que, devenue grande, elle avait manifesté ses qualités

6 Sur les récits de voyageurs grecs en Égypte pendant l'époque archaïque, voir C.-W. MÜLLER, "Fremdenfahrung und Eigenfahrung. Griechische Ägyptenreisende von Menelaos bis Herodot", Pbilologus 141 (1997), p. 200-214. Sur l'image de l'Égypte dans les œuvres littéraires grecques, voir Ch. Froidefont, Le mirage égyptien dans la littérature grecque d'Homère à Aristote, Aix-enProvence, 1971, spéc. p. 15-68.

7 Voir T.F.R.G. Braun, "The Greeks in Egypt", CAH, vol. III (1982²), part 3, p. 33-37.

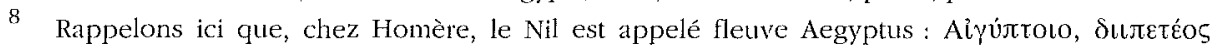

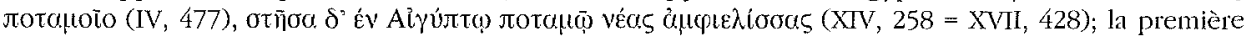

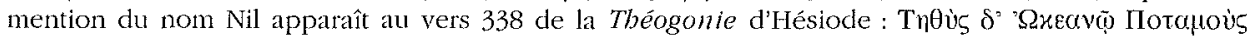

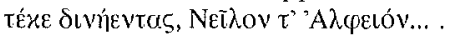

Od. $\mathrm{V}, 333-353$.

10 Voir p. ex. P. Plass, "Menelaus and Proteus", CJ 65 (1969), p. 104-108; Ch. A. VAN Duzer, Duality and Structure in the Iliad and Odyssey, Bern, Lang, 1996, p. 10-13.

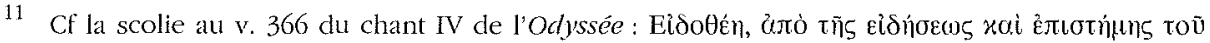

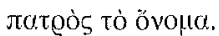

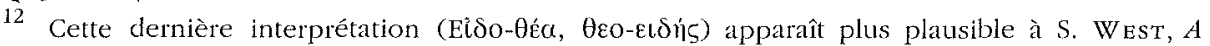
Commentary on Homer's Odyssey I, Oxford, 1990, p. 214.

13 Fr. 212 Nauck (= 5 Mette). 
prophétiques et qu'en vertu de ses qualités elle s'était appelée Théonoé ( $\Theta \varepsilon o v o ́ n)^{14}$. De sa préhistoire odysséenne, Eidô-Théonoé a conservé son activité bienfaisante à l'égard de Ménélas et d'Hélène. Euripide nous apprend - par la bouche d'Hélène - le nom de la mère d'Eidô et femme de Protée ${ }^{15}$. C'est Psamathé, ex-femme d'Aiacos à qui elle avait donné un fils, Phocos. Psamathé s'était transformée en phoque pour échapper à Aiacos et s'était servie du même talent pour échapper à Protée ${ }^{16}$. La mère d'Eidothéa n'est pas mentionnée dans l'Odyssée. On ne peut pas savoir si le mariage de Psamathé et d'Aiacos était connu d'Homère, mais on ne peut pas l'exclure puisque ces récits mythiques étaient au moins connus du poète des Cbants Cypriens. Du sort d'Eidô-Eidothéa dans la littérature post-homérique on sait du moins qu'Euripide avait de fortes raisons de dessiner l'intrigue de son Hélène telle qu'on la connaît. Son " alibi » littéraire repose sur l'Odyssée, sur Hésiode ${ }^{17}$, sur Hérodote ${ }^{18}$ et surtout sur la Palinodie de Stésichore ${ }^{19}$. Mais on peut se poser des questions sur la tradition que suivait de son côté Eschyle lorsqu'il a proposé l'histoire de Protée comme drame satyrique dans une tétralogie avec une unité thématique donnée, le retour et le sort d'Agamemnon. Or, nous savons, bien entendu, qu'il avait au moins une source, le chant IV de l'Odyssée. En avait-il d'autres?

\section{Protée}

Quoique le nom de Protée ait souvent été rapproché du mot $\pi \varrho \omega \tau o ́ v ~(\pi \varepsilon ́ \pi \varrho \omega \tau \alpha \iota)$ ou du thème du mot égyptien prouti $(=$ roi), il est plus convainquant de supposer qu'un auditeur hellénophone aurait tendance à l'associer au mot $\pi \varrho \tilde{u} \varsigma^{20}$. On aurait

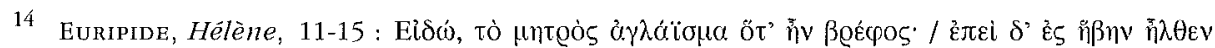

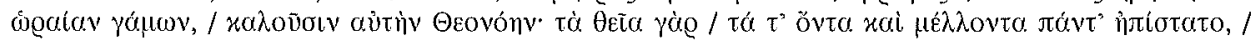

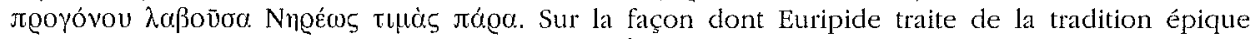
concernant le séjour de Ménélas et d'Hélène en Égypte, voir déjà F. JouAN, Euripide et les légendes des Chants Cjpriens, Paris 1966, spéc. p. 188-195, et aussi C.W. Marshall, "Idol Speculation: the Protean Stage of Euripides' Helen", $T E P 16$ (1995), p. 74-79.

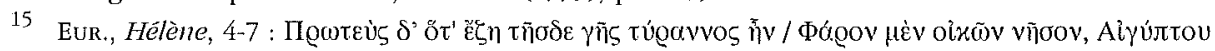

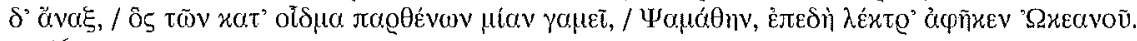

16 Pindare, Ném. V, 24.

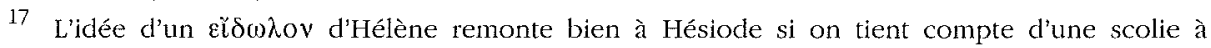
LyCOPHRON (Alex., 822) d'après laquelle il fut le premier à introduire ce fantôme.

18 II, 113-118.

19) Stésichore, fr. 16 Page; P. Ony. XXIX (1963), 2506, fr. 26a; voir aussi le commentaire de Page, p. 35-38, d'où l'on peut déduire que, selon Stésichore, Hélène avait accompagné Pâris en Égypte et que c'est précisement là-bas que le fameux fantôme s'est substitué à elle, grâce, notamment, à l'art des métamorphoses dont Protée était le maître; $c f$. L. GHaLi-KaHiL, Les enlèvements et le retour d'Hélène, Paris, 1955, p. 285-301; Marshald, l.c. (n. 14).

20 Sur les origines du mythe de Protée, sur l'antériorité ou la postériorité de son origine chalcidicienne, sur les interférences des éléments égyptiens et/ou bibliques par rapport au récit homérique, voir R. Goossens, "Un conte égyptien : Pharaon, roi des phoques", in Mélanges $F$. Cumont, Bruxelles, 1936, p. 715-722; K. O'NolaN, "The Proteus Legend", Hermes 88 (1960), p. 129138. Sur un certain aspect cosmologique ("Seinsprinzip") qu'on pourrait voir en Protée en tant que clivinité de l'eau, voir G. Dietz, "Okeanos und Proteus, Poseidon und Skamander: Urstorm, Meer und Fluss bei Homer", Symbolon 13 (1997), p. 35-58. Sur le récit de Protée par rapport aux structures typologiques de la narration odysséenne, voir M. STEInRück, "Der Bericht des Proteus", QUCC 42, 3 (1992), p. 47-60; sur la typologie des aspects associés à Protée et, en particulier, sur le thème du savoir et de la sagesse, voir J. Boulogne, "La leçon de Protée", Uranie 5 (1995), p. 9-32. Sur le devoir de compter les phoques de son troupeau, dont est chargé le Protée odysséen, et sur 
du mal a donner une réponse définitive à la question de savoir si l'idée de placer Protée en Égypte est une innovation d'Homère, étant donné que le rapport de Protée avec Chalcidice pourrait être très ancien ${ }^{21}$. De telles priorités narratives, comme la présence du Vieillard marin au littoral de Pharos, peuvent être bien servies par l'idée, d'ailleurs très fréquemment attestée dans l'épopée homérique, que les divinités maritimes peuvent à leur aise apparâttre dans n'importe quel point de leur espace naturel. Que ce soit ou non sous l'influence de l'Odyssée, Protée est considéré comme un roi d'Égypte par Hérodote (qui se réfère néanmoins à des sources égyptiennes) ${ }^{22}$ aussi bien que par Euripide dans l'Hélène.

Dans le système religieux constitué par les données de l'épopée homérique, protée est un des trois Vieillards de la mer, avec Nérée et Phorcys. Ces personnages divins de la mer, hormis la place honorable qu'ils conservent dans la plupart des généalogies mythiques ${ }^{23}$, ont également en commun le don de la métamorphose ${ }^{24}$; une parodie de métamorphose (déguisement en phoque) est d'ailleurs opérée par Ménélas pour tromper Protée. L'autre trait de Protée est le savoir prophétique. De ce point de vue, les révélations de Protée à Ménélas et les révélations de Tirésias à Ulysse sont comparables ou du moins homologues dans leur contexte narratif respectif ${ }^{25}$. Comme Tirésias à Ulysse, Protée clôt ses révélations à Ménélas avec la prédiction et la description de sa mort. Le retour au thème de la mort est la conclusion logique des révélations que Protée s'est engagé à faire en fonction du critère qu'il a lui-même introduit, à savoir la mort des chefs Achéens qui ont péri à la fin de leur nostos. Il est évident que, dans le cadre de ces révélations, le destin d'immortalisation qui attend Ménélas sert de contrepoint au destin mortel de son frère ${ }^{26}$, destin auquel le poète de l'Odyssée retourne par plusieures voies et à plusieurs reprises tout au long du poème. Dans les révélations de Protée concernant le sort d'Agamemnon, on peut également détecter l'usage plus complexe que fait Homère des traits mythiques et religieux

les diverses perspectives interprétatives ouvertes, au niveau contextuel et sémantique, par ce jeu d'arithmétique et de calcul, voir R. Piettre, "Les comptes de Protée", Métis 8, 1-2 (1993), p. 129-145.

21 De Wilamowitz (Hom. Unters. 27 A. 15, Der Glaube der Hellenen, I, p. 223) à S. West (o.c. [n. 12], p. 218), la plupart des chercheurs estiment que la persistance de la Chalcidique dans des sources postéricures à l'Odyssée (Lycophron, Alex., 115 sq.; Calumaque, Vict. Beven., 5-6, schol. Apollonius de Rhodes, I, 598; Apollodore, II, 5, 9; Virgile, Georg. IV, 390-391) et en dépit de l'influence exercée par l'odyssée, favorise l'hypothèse d'une origine nord-égéenne de Protée, tradition qu'Homère aurait modifiée en placant Protée en Égypte. Contra O'Nolan, l.c. (n. 20), p. $134 \mathrm{sq}$.

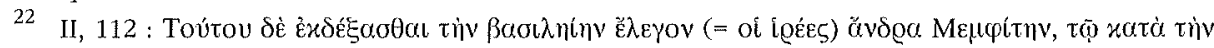

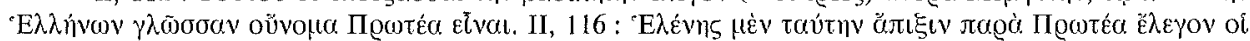

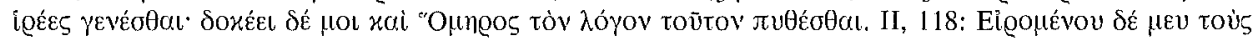

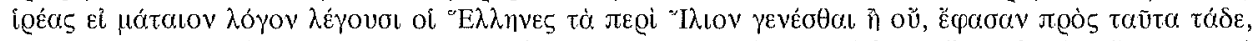

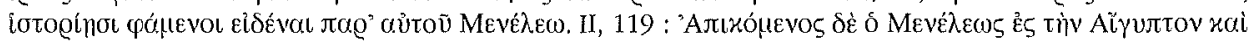

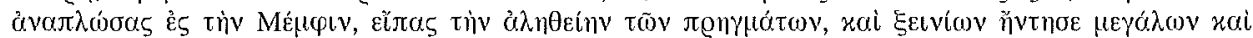

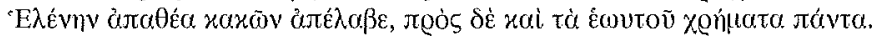

23 Un respect analogue est aussi attribué à d'autres divinités maritimes - non olympiennes comme Océan et Thétis.

24 Sur les métamorphoses des divinités maritimes, voir en particulier M. DeTienNe, J.-P. Vernant, Les ruses de l'intellignce. La métis des Grecs, Paris, 1974, p. 140; E. Vermeule, Aspects of Death in Early Greek Art and Poetry, Berkeley/Los Angeles, 1979, p. 190 sq.

25 Sur cette analogie, voir P. Plass, "Menelaus and Proteus", CJ 65 (1969), p. 104-108, spéc.

p. 104-105; cf aussi S. West, O.C. (n. 12), p. 218.

26 Voir également van Duzer, o.c. (n. 10), p. 38-42. 
attribués à Protée. Cet usage porte notamment sur la technique narrative d'Homère et sur le(s) présumé(s) poème(s) épique(s) pré-odysséen(s) non conservé(s) dont le thème était le retour et le sort d'Agamemnon ${ }^{27}$, poème(s) que le poète de l'Odyssée connaît bien, poème(s) qu'il se met probablement à dépasser avec son Odyssée. Les révélations faites par le divin et devin Protée répondent à des finalités pratiques et trouvent leur vérification manifeste au cours même de l'Odyssée $e^{28}$. Cependant, leur principale caractéristique constitutive est qu'elles ne sont pas tirées du domaine de la religion mais du domaine de l'épopée pré-homérique dont elles présupposent la connaissance. Si dans ce poème pré-odysséen sur le sort d'Agamemnon, dans cette présumée «descente des Atrides», il était question d'une action coordonnée de Ménélas et d'Agamemnon lors de leur retour en Grèce, l'errance de Ménélas en Égypte facilitait sans doute le meurtre d'Agamemnon par Égisthe. Au niveau narratologique, cette errance, odysséenne ou pré-odysséenne, est, de surcroît, extrêmement fonctionnelle. Quels éléments de ce poème pré-odysséen sur Agamemnon conserve Homère dans le récit odysséen de Protée, quels éléments il transforme, combien il s'avère traditionnel et combien innovateur, il est difficile de le préciser dans l'état présent de nos connaissances, tant que nous ignorons le contenu exact de cette importante source de l'odyssée. Mais il y a une chose qui reste certaine. L'usage génial du divin Protée dans l'Odyssée assure à son poète la plus haute crédibilité : ce qui, dans le récit de Protée, s'accorde avec les récits épiques antérieurs, trouve les auditeurs-connaisseurs consentants. Ce qui s'en éloigne s'appuie sur l'omniscience prophétique du divin Protée, tout en concordant avec la tendance constante de la poésie épique à attribuer son inspiration au niveau divin. Entretemps, la fiabilité du poète de l'Odyssée se renforce de plus en plus par la vérification des autres prédictions de Protée dans le poème même. La version narrative odysséenne est destinée à convaincre. Fidèle à sa mission narrative odysséenne, le Protée odysséen, dans sa métamorphose la plus efficace et subtile, n'est pas, au fond, invoqué pour apparaittre dans le rôle du dieu mais dans le rôle du poète à la fois pré-odysséen et odysséen.

Menelaos Christopoulos

Université de Patras

Département de Philologie

GR - 26500 Rio

27 L'idée qu'il ne s'agirait que de pures «traditions » mythiques sur le sort d'Agamemnon et pas nécessairement de poèmes épiques est, de toute évidence, à exclure; il est fort improbable que des traditions mythiques telles que le retour et la mort d'Agamemnon ou la vengeance d'Oreste, évoquées d'ailleurs fréquemment et presque obstinément dans l'Odyssée, aient pu circuler et se transmettre dans la Grèce de l'époque homérique par des voies autres que la poésie épique et que seuls les aèdes n'en aient pas parlé. Sur la dépendance du récit odysséen concernant le retour: d'Agamemnon par rapport à une éventuelle version non odysséenne du retour des deux Atrides, voir S. WEST, o.c. (n. 12) p. 224-226; sur le même sujet et, plus généralement, sur le problème très complexe des sources de l'Odyssée voir G. Danex, Epos und Zitat. Studien zu den Quellen der. Odyssee, Wien, 1998, p. 117-120.

28 Des neuf révélations énumérées dans le deuxième chapitre, sept trouvent leur vérification dans l'Odyssée elle-même. $1^{\text {re }}: I V, 581-583 ; 2^{\mathrm{e}}: \mathrm{IV}, 581-583 ; 3^{\mathrm{e}}: \mathrm{IV}, 538-540 ; 4^{\mathrm{e}}: \mathrm{IV}, 499-511 ; \mathrm{XI}, 405-$ $434 ; \mathrm{V}, 81-84 ; 140-144 ; 6^{\mathrm{e}}:$ XI, 405-434; $7^{\mathrm{e}}:$ III, 306-312; $8^{\mathrm{e}}: \mathrm{V}, 81-84$ 